Running Head: TRAINING VISUAL EXPLORATORY ACTIVITY

\title{
Using an Imagery Intervention to Train Visual Exploratory Activity in Elite Academy Football Players
}

\author{
Chris Pocock ${ }^{1,2}$
}

Matt Dicks ${ }^{2}$

Richard C. Thelwell ${ }^{2}$

Michael Chapman ${ }^{2}$

Jamie B. Barker ${ }^{3}$

Author Note

${ }^{1}$ Expert Performance and Skill Acquisition research group, School of Sport, Health and Applied Science, St Mary's University, Twickenham, U.K.

${ }^{2}$ Department of Sport and Exercise Science, University of Portsmouth, U.K.

${ }^{3}$ Centre for Sport, Health and Exercise Research, Staffordshire University, U.K.

Correspondence concerning this article should be addressed to Chris Pocock, School of Sport, Health and Applied Science, St Mary's University, Twickenham, TW1 4SX. Tel: 0208240 8243; Email: Chris.Pocock@stmarys.ac.uk

\footnotetext{
Accepted for publication in Journal of Applied Sport Psychology on $18^{\text {th }}$ October, 2017
} 


\section{Abstract}

Football players adapt their movements to opportunities within the surrounding environment by engaging in Visual Exploratory Activity (VEA) to pick-up information. This study adds to the extant literature by using a six-week PETTLEP imagery intervention to train VEA and improve performance with the ball. A single-case, multiple-baseline across participants' design was conducted with five elite academy football players. Results indicated that a PETTLEP imagery intervention improved VEA, particularly in center midfielders. Additionally, indications of improvements in performance with the ball were present within some participants. Future researchers could examine the processes underpinning VEA to enhance applied interventions for 31 this skill.

Key words: Visual exploratory activity, PETTLEP imagery, affordances, decision-making 


\section{Using an Imagery Intervention to Train Visual Exploratory Activity in Elite Academy}

\section{Football Players}

Expertise in fast-ball sports such as cricket, tennis, and football is predicated on the control of accurate and skillful movements under strict spatiotemporal constraints. Research indicates that successful performance in team sport necessitates that players constantly adapt their actions relative to changes in the environment (Araújo, Davids, \& Hristovski, 2006). For example, when in possession of the ball, football players are required to decide whether they should pass or run with the ball. With every unfolding moment, information in the environment (e.g., position of teammates and opponents) will alter, inviting a new set of possible actions. Importantly, decisions will emerge relative to the accurate perception of what the environment offers a performer given his/her movement capabilities (i.e., affordances: Fajen, Riley, \& Turvey, 2009; Gibson, 1979). In this regard, accurate perceptual-motor control is underpinned by the education of attention towards task relevant information that is scaled to a performer's movement capabilities (Dicks, Davids, \& Button, 2010; Orth, Davids, Araújo, Renshaw, \& Passos, 2014).

Research proposes that decision-making in team sports consists of transitions in courses of action that reflect the use of available information in the environment, rather than a set of discrete choices at separate decision points (Araújo et al., 2006). Theoretically, such a proposal is reconciled by Gibson's (1979) ecological approach, which places emphasis on the reciprocal nature of perception-action and the importance of studying the pick-up of information as an active process, which encompasses the mobile body (for reviews, see Fajen et al., 2009; van der Kamp, Rivas, van Doorn, \& Savelsbergh, 2008). In support of such assertions, research indicates that sport performers engage in VEA to prospectively control actions and adapt to the dynamic and emergent nature of sport situations (Jordet, 2005). Specifically, for behavior in team ball 
sports, Jordet (2005) defined exploratory activity as "A body and/or head movement in which the player's face is actively and temporarily directed away from the ball, seemingly with the intention of looking for teammates, opponents or other environmental objects or events, relevant to the carrying out of a subsequent action with the ball" (p.143). The importance of such behavior is thought to support skilled performance as analysis of elite football players indicates that a higher frequency of VEA before receiving the ball is reflective of a higher forward passing accuracy (Jordet, Bloomfield, \& Heijmerikx, 2013).

Engagement in exploratory actions is thought to be critical for the acquisition of perceptual-motor control (Adolph, Eppler, Marin, Weise, \& Clearfield, 2000) as this supports the attunement of actions to changing properties of the environment (Reed, 1996). For example, in a recent climbing study, Seifert and colleagues revealed that behavioral exploration during practice led to increased climbing fluency in a transfer test (Seifert, Boulanger, Orth, \& Davids, 2015). Such evidence highlights that exploratory or variable actions can play a performatory role during the control of action (Stoffregen \& Mantel, 2015). Specifically, prospective or anticipatory control has been conceptualized as "exploration from a distance" (Adolph et al., 2000, p.447), whereby ongoing movements are adapted to changes in the environment to generate information or possibilities for the control of subsequent actions. In the context of sport, initial findings have highlighted the importance of anticipatory control where the availability of advance visual information is used to control ongoing actions, such as a goalkeeper's movements when facing a penalty kick (e.g., Dicks, Button, \& Davids, 2010).

Given that anticipatory control is critical to performance in sport, a number of studies have investigated whether this facet of skilled performance can be enhanced through training, in particular, via video-based simulations (for a review, see Dicks, van der Kamp, Withagen \& 
Koedijker, 2015). Dicks and colleagues highlighted that although a large number of video training studies have been conducted (e.g., Williams, Ward \& Chapman, 2003), the vast majority have not included transfer tests to examine whether changes in performance on video-based tasks of anticipation and decision-making lead to commensurate improvements during on-field improvements. An example of previous research that has examined on-field performance is the study conducted by Jordet (2005), who investigated whether an imagery intervention combined with observation of video footage could improve the decision-making of elite football players. Following the intervention, the players were found to increase the amount of VEA, although there were no improvements in decision-making. The absence of performance improvements exhibited were explained by a 'ceiling effect' as the participants were playing at an elite, international level. Despite this finding, it has been proposed that an imagery intervention, as used by Jordet (2005), may support the development of perceptual skill (e.g., Smeeton, Hibbert, Stevenson, Cumming, \& Williams, 2013).

An imagery intervention that has received much attention in the sport psychology literature is the PETTLEP model (for a review, see Wakefield, Smith, Moran, \& Holmes, 2013). The PETTLEP model (Holmes \& Collins, 2001) emphasizes that imagery interventions should be individualized and aim to simulate a performer's execution situations, with emphasis on experiences associated with movements and their associated emotional consequences. The seven components of the PETTLEP model (Physical, Environment, Task, Timing, Learning, Emotion and Perspective) can be used individually or in combination to increase the effectiveness of an imagery intervention (Holmes \& Collins, 2001). Interventions using adult participants have supported the use of PETTLEP imagery in improving performance of motor skills (e.g., Smith, Wright, Allsopp, \& Westhead, 2007; Smith, Wright, \& Cantwell, 2008). A study of junior 
103

104

105

106

107

cricketers found that imagery rehearsal mimicking physical practice via the PETTLEP

framework, combined with video training, led to improvements in performance on a video-based anticipation task (Smeeton et al., 2013). However, an important limitation of Smeeton et al.'s work was the absence of an on-field transfer test, meaning that it remains unknown whether the PETTLEP intervention enhanced anticipation during actual batting performance. Specifically, recent studies in the anticipation literature have highlighted that the processes underpinning anticipation skill are different for video-based judgement tasks and real-time interception tasks (e.g., Dicks et al., 2010). It therefore follows that an important requirement in contemporary perceptual learning studies is to include on-field transfer tests (Dicks et al., 2015). Furthering the extant literature (Smeeton et al., 2013) in this present study we examine whether a combined video and imagery intervention leads to improvements in anticipatory control when performance is assessed beyond the laboratory. Specifically, we examined whether a combined PETTLEP imagery and video training intervention improved VEA and decisionmaking in elite academy level football players. A secondary aim was to study whether any increase in VEA enhances performance with the ball in match situations (cf. Jordet, 2005). Significantly, we address calls to examine the benefits of a perceptual training intervention beyond a simulated laboratory setting (see Dicks et al., 2015) and examine whether the previous lack of improvement in performance accuracy are attributable to ceiling level performance as reported by Jordet (2005). In this instance, we hypothesized that increased VEA will lead to improved performance (decision-making) with the ball (Jordet et al., 2013) for elite academy football players.

\section{METHOD}

\section{Participants}


Five male participants were recruited from an under 18 elite (professional) UK academy

football team. All participants were aged 16 and 17 and provided informed written consent before taking part in the study. The five participants were selected based on their position in the team's formation; only midfielders and forwards were recruited after a discussion with the team's coach regarding position-specific responsibilities. This was attributed to the hypothesis that to support successful action, midfielders and forwards need to engage in VEA prior to receiving the ball (Jordet et al., 2013). Participants 1 and 3 played as central midfielders, Participants 2 and 4 played as wide midfielders and Participant 5 played as a center forward. The study was approved by the lead author's University's ethics committee. Experimental Design

A single-case, staggered multiple-baseline across-participants design was used to explore intervention effects for each participant. The multiple-baseline design remains the most effective design for exploring intervention effects in sport psychology research (see Barker, Mellalieu, McCarthy, Jones, \& Moran, 2013). In this design, the introduction of the intervention typically takes place when a stable baseline of the dependent variable is achieved, or performance moves in a direction opposite to that expected following treatment (Hrycaiko \& Martin, 1996). Each participant acts as their own control, thus allowing for comparison at a within-subject level between baseline and post-intervention phases (Gage \& Lewis, 2013).

The order of participants taking part in the intervention was determined by the most stable baseline measures across the first five matches (equivalent to four weeks; Carr, 2005). The present study lasted 21 weeks and the intervention was introduced to Participant 1 in week 5, which is in line with previous research (e.g., Wakefield \& Smith, 2011). The intervention lasted for six weeks for each participant, except for Participant 4 who completed a seven-week 
intervention as a minor injury prevented him from training for one week. Participants completed one supervised imagery session per week and were encouraged to complete two individual sessions per week (recorded using an imagery diary; Jordet, 2005) as using PETTLEP imagery for three times a week (Wakefield \& Smith, 2009) for six weeks (Finn, Grills, \& Bell, 2009) has been suggested as an effective frequency of intervention.

Procedure

Data Collection. Recordings of 19 matches in the team's season were analyzed to collect performance data. Ten randomly selected situations - less than ten if a participant was used as a substitute - were collected from each match. A situation was collected if the participant was in control of the ball and visible on camera for at least 5 seconds before receiving the ball.

Intervention. The imagery intervention consisted of the participants imagining themselves in a match situation and engaging in VEA before receiving the ball. The principal investigator and the football club's academy sport psychologist in training led the intervention. Prior to starting the intervention, the coaching staff were consulted to outline each participant's position in the team's formation and expectations of the position in the team. Specifically, the coaching staff provided information regarding the team's playing philosophy to ensure that the intervention was individualized and underpinned the team's tactics and style of play. Participants completed the MIQ-R (Hall \& Martin, 1997) as part of the first intervention session to provide baseline imagery ability scores. The following intervention sessions focused on upcoming match situations using personalized scripts that were developed with the feedback of the coaching staff. Participants also had the opportunity to watch video sequences of their VEA in a recent match situation before engaging in imagery. The imagery script instructed participants to engage in VEA as if they were searching for information away from the ball but 
172 the participant decided the pattern of play, for example: "As the ball is travelling towards you,

173 imagine checking for team mates in advanced positions. Imagine controlling the ball. You have

174 the ball, now I want you to imagine what you will do with the ball”. The participants were

175 instructed to image three match scenarios and actions in each session. The imagery scripts are

176 available upon request from the first author.

177 Components of the PETTLEP model (Holmes \& Collins, 2001) were used in the

178 development of the script. The Physical and Perspective components of the PETTLEP model

179 were manipulated by instructing participants to 'step inside' the video with their eyes closed as if

180 they were preparing to receive the ball (Smeeton et al., 2013). To use elements of the

181 Environment component, participants were told to feel the pitch beneath them and imagine the

182

183

184

185

186

187 weather of the upcoming match. Task and Timing components were manipulated by allowing the participant to 'play' the ball and this encouraged the participants to think about future action opportunities (Jordet, 2005; Smeeton et al., 2013). Discussions with each participant after imagery revealed the outcome of the imagined scenario (e.g. team scoring a goal), which engages the Emotion component (Smith et al., 2007).

During the intervention, participants were asked to complete an imagery diary to record the number of individual imagery sessions performed (Wright \& Smith, 2009) and to note down any difficulties that they experienced while performing imagery (Wakefield \& Smith, 2011). Participants were encouraged to use their imagery script once individually and to observe one televised football match every week. The purpose of watching a game was for the players to observe the VEA of an elite player in their position and to make notes of their observations in their imagery diary. Throughout the six weeks, Participants 1 and 5 completed seven individual sessions, Participant 2 completed five, Participant 3 completed six, Participant 4 completed four. 
Once the participants had gained a clear understanding of the scripts, instructions shifted to the use of action lexicon such as 'search' and 'scan' (Jordet, 2005). Furthermore, videos were removed from the sessions. The rationale behind this shift was to ensure that script was easier to practice following the completion of the intervention and such imagery applied the Learning element of the PETTLEP model (Holmes \& Collins, 2001).

Dependent Measures

Visual Exploratory Activity. The measure of VEA was based on Jordet's (2005) definition of exploratory search. VEA was calculated by recording the number of explorations in a situation and dividing it by the number of seconds of that situation. The final five seconds before receiving the ball was defined as the online scanning period and any footage visible of the participant 10-5 seconds before receiving the ball was defined as the extended scanning period. Action Completion Rate. An action was considered successful if it resulted in continued possession of the ball for the team. Action completion rate was calculated by dividing the number of successful actions by the number of situations selected for analysis in a match. Direction of Actions. The number of forward actions and actions that were performed in a different direction to the direction of receiving the ball were recorded in each match. Actions were analyzed using Kinovea and were recorded as 'performed in a different direction' if the angle between the pass to the participant and the participant's action was greater than $90^{\circ}$. Decision-Making. Using action completion rate in isolation would not differentiate between a successful risky forward pass and a successful short backwards pass. Therefore, situations were evaluated by two coaches (with UEFA B qualifications) on a scale of 1-7 and were typically scored as follows; 1-3 if the player in possession unnecessarily lost the ball, 4 reflected intermediate performance and 5-7 for penetrating or efficient actions (Jordet, 2005). 

Smith, 2011), imagery ability was scored using a 7-point Likert scale in response to the MIQ-R (Hall \& Martin, 1997). The MIQ-R is an eight-item questionnaire that assesses one's ability to perform visual and kinesthetic imagery. Participants were asked to rate the ease or difficulty of imaging the movement ranging from 1 (very hard to see/feel) to 7 (very easy to see/feel). thoughts on the progress of the intervention (see Page \& Thelwell, 2013). Participants and the coach engaged in post-intervention interviews to explore the effectiveness of the intervention and its effects (Barker et al., 2013; Page \& Thelwell, 2013). The interviews allowed open-extended answers to be given based on the outcomes and experiences of the intervention. Procedural Reliability

To ensure that each participant was treated equally, post-intervention scores were not and structured nature of the intervention protocol ensured consistency of delivery across all participants (Barker et al., 2013).

Data Analysis collected in each match. Kinovea was used to analyze situations and collect quantitative performance data as this software program had the capacity to clip matches and a zoom tool to analyze VEA. Decision-making was analyzed by two qualified coaches (UEFA B Licence) using the 1-7 scale (Jordet, 2005). The final data was analyzed using visual graph inspection. Visual graph analysis with comparison of mean values is recognized as an accepted alternative to statistical techniques in SCD's, with six features of the graphic display that can be interpreted: 
241 (a) level, (b) trend, (c) variability, (d) immediacy of the effect, (e) overlap, and (f) consistency of 242 data pattern across similar phases (Gage \& Lewis, 2013). A paired-samples T-test was used to compare baseline and post-intervention imagery ability.

\section{RESULTS}

\section{Visual Exploratory Activity}

Post-intervention improvements in the mean level of VEA during the extended scanning period (Figure 1) are indicated for Participants (P) 2, 4 and 5. These improvements should be considered with some caution, as there are two or more overlapping data points when comparing post-intervention to baseline measures for P2, P4 and P5, all of which were recorded two weeks after completion of the intervention. During the extended scanning period, P1 showed initial indications of improvements in VEA at the start of the intervention although this decreased in week 6 and post-intervention. There appeared to be no intervention effect on P3 in the extended scanning period. P1 and P3 demonstrated improvements in the mean level of VEA during the online scanning period (Figure 1). P1 demonstrated the strongest indications of an intervention effect, as there are no overlapping data points when comparing post-intervention to baseline. Furthermore, confidence that an effect was observed for P1 is enhanced by the increase in VEA at the introduction of the intervention. However, for $\mathrm{P} 3$ there are overlapping data points with baseline measures in the three immediate weeks following the intervention. The data for P2, P4 and P5 is variable throughout.

$<<<<<<<<<<<<<<<$ Insert Figure 1 about here $>>>>>>>>>>>>>>>$

\section{Performance with the ball}

Participants improved (P1, P3, P4, and P5) or maintained (P2) their mean level of action completion rate in post-intervention compared to baseline (Figure 2). Whilst there was noticeable 
variability in the action completion rate of all participants, P1 and P3 showed the largest postintervention increases in action completion rate. The strongest indication of an intervention effect is present in P1's data with no overlapping points and a substantial increase in average post-intervention action completion rate compared to the stable baseline. The improvements in P3's action completion rate should be considered with caution, as there are overlapping data points with baseline measures in the immediate two weeks following completion of the intervention. Increases were demonstrated in P4 and P5 with variable data but there was no assumed intervention effect on P2. No intervention effects were reported for number of forward actions or actions performed in a different direction to the direction of receiving the ball $\left(>90^{\circ}\right)$. $<<<<<<<<<<<<<<$ Insert Figure 2 about here $>>>>>>>>>>>>>>>$

\section{Decision-Making}

Post-intervention decision-making scores were at a higher level compared to baseline for all participants (Figure 3). The greatest increase was observed for P4 with a distinct increase in scores from baseline reflecting the positive trend in the final weeks of the intervention and the immediate week following the intervention. Data should be considered with caution as there are overlapping data points when comparing post-intervention to baseline measures for P1, P2 and P3 in the immediate week following the intervention. P5 improved his level of decision-making but there was one overlapping data point post-intervention.

$<<<<<<<<<<<<<<<$ Insert Figure 3 about here $>>>>>>>>>>>>>>>$

\section{Imagery Ability}

Participants significantly improved imagery ability $(t(4)=-3.936, p=.017, d=2.47)$ when comparing post-intervention to baseline measures for kinesthetic scenarios of the MIQ-R 
286

287

(Hall \& Martin, 1997), but there was no significant improvement for visual scenarios $(t(4)=$ 2.039, $p=.111, d=0.97$; Table 1).

Insert Table 1 about here $>>>>>>>>>>>>>>>$

\section{Social Validation}

Social validation data collected throughout the intervention revealed that participants were satisfied with the organization of the intervention, the content of the imagery sessions and that the intervention was helping to improve VEA. On completion of the study, social validation interviews were conducted with all participants and the team's coach. In response to a question on the effect of the intervention on performance, P1 stated, "I've noticed my scanning has improved every game... so I think it's clear that the six-week intervention has worked”. P3 stated, "the sessions were acceptable and I now know how important it (VEA) is in my game". P4 identified that he "didn't really notice he was scanning before, but now I'm thinking more about scanning in the game situation and in training". The use of imagery was also discussed with participants, with P2 recalling, "Sometimes those scenarios come out in games. Almost like you're thinking about it before it actually happens, which is good". P2 added: "When it happens in the game after you've imaged it, I think it's quite interesting because you know that you've thought about that before". The individual imagery diary was discussed with P5, who stated, "I analyze players more often and when watching a match now, I'll watch a single player in my position straight away".

A post-intervention interview with the team's coach provided an external view of the intervention and the impact that it had on the participants. The coach indicated, "There was an impact, particularly on the center midfield players and especially Participant 1 during training and matches". This was attributed to the intervention being "the most appropriate for players in a 
central position" as they "require $360^{\circ}$ awareness". The coach was very positive on the performance impact that the intervention had, stating, "The quality and frequency of the scans certainly improved". The coach felt that the intervention and imagery scripts were suitable for the purpose of the study and were manageable alongside the regular training of participants.

\section{DISCUSSION}

The purpose of the present study was to examine whether a combined PETTLEP imagery and video training intervention can improve VEA and decision-making in elite academy level football players. We aimed to examine the benefits of a perceptual training intervention beyond a laboratory setting and whether the previous lack of improvement in performance can be attributable to ceiling level performance (Jordet, 2005). Following previous research (Jordet, 2005), we hypothesized that the imagery intervention would produce an increase in VEA and improve performance with the ball in match situations as a higher level of VEA has been shown to be reflective of improved performance (Jordet et al., 2013). The imagery intervention of Jordet's (2005) study demonstrated improvements in VEA of elite football players with international caps, but recorded no improvements of performance with the ball. The participants of the present study were academy level football players and thus, we expected, had scope for improvement in performance with the ball. The present study lends partial support to our hypothesis and is consistent with past research in that the use of an imagery intervention enhanced VEA. However, there were no consistent improvements in performance with the ball across all participants (Jordet, 2005). The strongest indications of improvements in VEA were displayed in center midfielders (Participants 1 and 3) and supported by the view of the team's coach that center midfielders require " $360^{\circ}$ awareness" (see Figure 1). Although there were smaller indications of improvements in VEA for wide midfielders (Participants 2 and 4), and the 
team's center forward (Participant 5), decision-making of all participants improved when comparing post-intervention to baseline (see Figure 3). Action completion rate data was variable, although substantial improvements are indicated for Participant 1 (see Figure 2), and there were no intervention effects on action completion rates for all participants when playing the ball forward or in a different direction to which the ball was received.

Our results indicate that exploratory behavior appears to play a critical role in perceptionaction across extended and online time-scales. Without anticipatory control, an individual's action would be reduced to mere reaction, which would not suffice in fast-paced sport environments (Fajen et al., 2009). In our present study, the improvements reported in online VEA appear to play an important role in decision-making performance. Specifically, VEA in the final five seconds before receiving the ball appeared to enable participants to exploit information that supported subsequent actions with the ball. Such suggestion is in line with previous findings, which indicate that online adjustments ensure that actions can be performed within a performer's action capabilities (Dicks, Davids et al., 2010; Orth et al., 2014).

Increased VEA may lead to the search for more information, which would present further potential opportunities for action (affordances). Perception of opportunities for action will be grounded in a football player's physical and technical capabilities (Fajen et al., 2009). Thus, a football player can be exposed to more information in the environment, in the sense that they are relying on an informational variable, but this extra information may not be calibrated to their action capabilities (Dicks et al., 2015; Fajen et al., 2009). It is possible that for complex perceptual-motor skills that take place within dynamic sport situations, this period of (re)calibration may require a long duration of practice and hence why the more stable changes of performance with the ball are observed for participants with the longer post-intervention phases. 
Future work may therefore benefit from examining performance improvements over a longer post-intervention period. Although post-intervention improvements were reported for decisionmaking (see Figure 3), participant performances were typically recorded as being at an intermediate level (4). Strong indications of an improvement in performance with the ball were noticeable in isolated cases, however lack of widespread improvements across participants can potentially be attributed to the design of the PETTLEP imagery, which emphasized simulations of VEA but not necessarily decision-making (see also, Jordet, 2005). Overall, the video and imagery intervention may have led to a positive change in online VEA, however this same intervention did not transfer to the control of opportunities for action for all participants. Although the imagery intervention produced post-intervention improvements in imagery ability for all participants (see Table 1), there were only a minority of participants who displayed clear improvements in performance with the ball. This finding suggests that the combined video and imagery intervention may have led to improvements in vision for perception but had no effect on vision for perception-action (van der Kamp et al., 2008; Dicks et al., 2015). It has been suggested that PETTLEP imagery scripts may develop perceptual-motor skill associated with ventral system (vision for perception) processes (Holmes \& Collins, 2001; Wakefield et al., 2013), which act on a longer timescale than the online control of actions (Madary, 2011; van der Kamp et al., 2008). In contrast, dorsal system (vision for action) processes play a fundamental role in anticipation (Madary, 2011) and are underpinned by information exploited during movement control (van der Kamp et al., 2008). That is, it is plausible that there were no substantial improvements with the ball observed in the present study as the PETTLEP intervention may only primarily support processes associated with vision for perception (ventral system) and not vision for action (dorsal system). Complementary to views highlighted 
elsewhere (e.g., Wakefield et al., 2013) future researchers would benefit from the exploration of the time-scales of perceptual-motor control that are best supported by imagery interventions. The design of the present study enabled social validation to be collected during the intervention through fortnightly semi-structured interviews (Page \& Thelwell, 2013). Participant 4 represents an example of the usefulness of participant interviews during the intervention phase, as this allowed the adaptation of his intervention to seven weeks due to injury. Researchers using single-case designs (SCD) should implement regular social validation to further individualize the intervention for respective participants. Consistent with recent SCD research (e.g., Turner \& Barker, 2013), a post-intervention interview was administered with the team's coach. The coach's positive comments on the development of the participants reinforce the effectiveness of the intervention. The strongest suggestions of an intervention effect are reflected in Participant 1, which was supported by the coach's interview. Reporting the views of a coach adds to the VEA literature that has previously only reported the views of participants (Jordet, 2005).

Social validation data indicated that the coach recognized that the frequency and quality of scans (VEA) had improved. Improvements in perceptual-motor skill are likely to reflect enhanced attunement of exploratory behaviors to task demands (Reed, 1996). Thus, ongoing VEA may become unnecessary if players are attuned to information that will support subsequent perception-action (Dicks et al., 2015). Given previous findings (Jordet et al., 2013), such interpretation appears most appropriate for wide positions as there were minimal postintervention effects on VEA in these players (Participants 2 and 4). Nevertheless, both wide midfielders (right midfielder and left midfielder) improved decision-making when comparing post-intervention performance to baseline. This suggests that the imagery intervention improved the quality of VEA (albeit at an intermediate level) due to the wide midfielders learning where 
401

402

403

404

and when to scan. Thus, analogous to findings of differences in the physiological demands of respective football player positions (e.g., Gonçalves, Figueira, Maçãs, \& Sampaio, 2014), our study indicates that perceptual-motor skill demands appear to vary between playing positions. Future researchers should consider further examination of how VEA is adapted relative to changes in player abilities (e.g., Dicks, Davids et al., 2010) and player position. Moreover, further researchers should accommodate individual differences in perceptual learning interventions (Dicks et al., 2015). In this regard, the individualized nature of the PETTLEP intervention (Wakefield et al., 2013), means that such position- and player-specific requirements can be catered for during training. Despite the significant scope available for future researchers, the current study provides practical recommendations for coaches within similar settings, namely; (i) the need for an awareness of the importance of VEA in the development of decision making and specific performance outcomes, particularly in players within certain playing positions, (ii) the importance of integrating perceptual skill training interventions alongside those of a technical and physical nature to develop VEA behaviors, (iii) and the potential efficacy of imagery and video training as a supplement to physical practice to facilitate such development.

The results from our study lend support to previous research (Jordet, 2005) and show that a PETTLEP based imagery intervention can produce improvements in VEA of elite academy level football players. Future researchers should seek to understand the time-scales of perceptualmotor control that are best facilitated by imagery interventions. Moreover, future researchers could examine how differences in player position and abilities influence VEA. Finally, the present study was one of the first to use regular social validation with young athletes in a SCD (Page \& Thelwell, 2013) and therefore researchers may consider the use of ongoing social validation to tailor the intervention to the participant's needs and to explore intervention efficacy. 


\section{References}

Adolph, K. E., Cole, W. G., Komati, M., Garciaguirre, J. S., Badaly, D., Lingeman, J. M., Chan, G. L. Y., \& Sotsky, R. B. (2012). How Do You Learn to Walk? Thousands of Steps and Dozens of Falls per Day. Psychological Science, 23(11), 1387-1394.

Adolph, K. E., Eppler, M. A., Marin, L., Weise, I. B., \& Wechsler Clearfield, M. (2000). Exploration in the service of prospective control. Infant Behavior and Development, $23(3), 441-460$.

Araújo, D., Davids, K., \& Hristovski, R. (2006). The ecological dynamics of decision making in sport. Psychology of Sport and Exercise, 7(6), 653-676.

Barker, J. B., Mellalieu, S. D., McCarthy, P. J., Jones, M. V., \& Moran, A. (2013). A review of single-case research in sport psychology 1997-2012: Research trends and future directions. Journal of Applied Sport Psychology, 25(1), 4-32.

Carr, J. E. (2005). Recommendations for reporting multiple-baseline designs across participants. Behavioral Interventions, 20(3), 219-224.Dicks, M., Button, C., \& Davids, K. (2010). Availability of advance visual information constrains association-football goalkeeping performance during penalty kicks. Perception, 39(8), 1111-1124.

Dicks, M., Davids, K., \& Button, C. (2010). Individual differences in the visual control of intercepting a penalty kick in association football. Human Movement Science, 29(3), 401411.

Dicks, M., van der Kamp, J., Withagen, R., \& Koedijker, J. (2015). Can we hasten expertise by video simulations? Considerations from an ecological psychology perspective. International Journal of Sport Psychology, 46(6), 587-607. 
Fajen, B. R., Riley, M. A., \& Turvey, M. T. (2009). Information, affordances, and the control of action in sport. International Journal of Sport Psychology, 40, 79-107.

Finn, J., Grills, A., \& Bell, D. (2009). A comparison of PETTLEP imagery, physical practice and their combination in the facilitation of non-dominant leg kicking accuracy. International Research in Science and Soccer, 177-187.

Gage, N. A., \& Lewis, T. J. (2013). Analysis of effect for single-case design research. Journal of Applied Sport Psychology, 25(1), 46-60.

Gibson, J. J. (1979). The Ecological Approach to Visual Perception. Boston: Houghton Mifflin.

Gonçalves, B. V., Figueira, B. E., Maçãs, V., \& Sampaio, J. (2014). Effect of player position on movement behaviour, physical and physiological performances during an 11-a-side football game. Journal of Sports Sciences, 32(2), 191-199.

Hall, C. R., \& Martin, K. A. (1997). Measuring movement imagery abilities: A revision of the movement imagery questionnaire. Journal of Mental Imagery, 21, 143-154.

Holmes, P., \& Collins, D. J. (2001). The PETTLEP approach to motor imagery: A functional equivalence model for sport psychologists. Journal of Applied Sport Psychology, 13(1), $60-83$.

Hrycaiko, D., \& Martin, G. L. (1996). Applied research studies with single-subject designs: Why so few? Journal of Applied Sport Psychology, 8(2), 183-199.

Jordet, G. (2005). Perceptual training in soccer: An imagery intervention study with elite players. Journal of Applied Sport Psychology, 17(2), 140-156.

Jordet, G., Bloomfield, J., \& Heijmerikx, J. (2013). The hidden foundation of field vision in English Premier League (EPL) soccer players. Sport Analytics Conference (pp. 1-6). Boston: Mit Sloan. 
469

470

471

472

473

474

475

476

477

478

479

480

481

482

483

484

485

486

487

488

489

490

Madary, M. (2011). The dorsal stream and the visual horizon. Phenomenology and the Cognitive Sciences, 10(4), 423-438.

Orth, D., Davids, K., Araújo, D., Renshaw, I., \& Passos, P. (2014). Effects of a defender on runup velocity and ball speed when crossing a football. European Journal of Sport Science, 14(1), 316-323.

Page, J., \& Thelwell, R. (2013). The value of social validation in single-case methods in sport and exercise psychology. Journal of Applied Sport Psychology, 25(1), 61-71.

Reed, E. S. (1996). Encountering the world: Toward an ecological psychology. New York: Oxford University Press.

Seifert, L., Boulanger, J., Orth, D., \& Davids, K. (2015). Environmental design shapes perceptual-motor exploration, learning, and transfer in climbing. Frontiers in Psychology, $6,1819$.

Smeeton, N. J., Hibbert, J. R., Stevenson, K., Cumming, J., \& Williams, A. M. (2013). Can imagery facilitate improvements in anticipation behavior? Psychology of Sport and Exercise, 14(2), 200-210.

Smith, D., Wright, C., Allsopp, A., \& Westhead, H. (2007). It's all in the mind: PETTLEP-based imagery and sports performance. Journal of Applied Sport Psychology, 19(1), 80-92.

Smith, D., Wright, C. J., \& Cantwell, C. (2008). Beating the bunker: The effect of PETTLEP imagery on golf bunker shot performance. Research Quarterly for Exercise and Sport, 79(3), 385-391.

Stoffregen, T. A., \& Mantel, B. (2015). Exploratory movement and affordances in design. Artificial Intelligence for Engineering Design, Analysis and Manufacturing, 29, 257-265. 
491

492

493

494

495

496

497

498

499

500

501

502

503

504

505

506

507

508

509

510

511

512

513

Turner, M., \& Barker, J. (2013). Examining the efficacy of Rational-Emotive Behavior Therapy (REBT) on irrational beliefs and anxiety in elite youth cricketers. Journal of Applied Sport Psychology, 25(1), 132-148.

van der Kamp, J., Rivas, F., van Doorn, H., \& Savelsbergh, G. (2008). Ventral and dorsal contributions in visual anticipation in fast ball sports. International Journal of Sport Psychology, 39(2), 100-130.

Wakefield, C., \& Smith, D. (2009). Impact of differing frequencies of PETTLEP imagery on netball shooting performance. Journal of Imagery Research in Sport and Physical Activity, 4(1), 1-12.

Wakefield, C., \& Smith, D. (2011). From strength to strength: A single-case design study of PETTLEP imagery frequency. Sport Psychologist, 25(3), 305-320.

Wakefield, C., Smith, D., Moran, A. P., \& Holmes, P. (2013). Functional equivalence of behavioural matching? A critical reflection on 15 years of research using the PETTLEP model of motor imagery. International Review of Sport and Exercise Psychology, 6(1), 105-121.

Williams, A. M., Ward, P., \& Chapman, C. (2003). Training perceptual skill in field hockey: Is there transfer from the laboratory to the field? Research Quarterly for Exercise and Sport, 74(1), 98-103.

Wright, C., \& Smith, D. (2009). The effect of PETTLEP imagery on strength performance. International Journal of Sport and Exercise Psychology, 7(1), 18-31. 
515 Figure 1: Pre- and post-intervention VEA in the extended and online scanning periods $\mathrm{s}^{\mathrm{a}}$.

$516{ }^{\text {a }}$ Missing data points are due to injury or non-selection. Participant 4 sustained a minor injury

517 during the intervention period and the intervention was prolonged to seven weeks for this 518 participant.

519 Figure 2: Pre- and post-intervention action completion rates.

Figure 3: Pre- and post-intervention decision-making scores.

Table 1: Baseline and post-intervention imagery ability scores. 
533

534

535

536

537

538

539

540

541

542

543

544

545

546

547

548

549

550

551

\section{Figures}


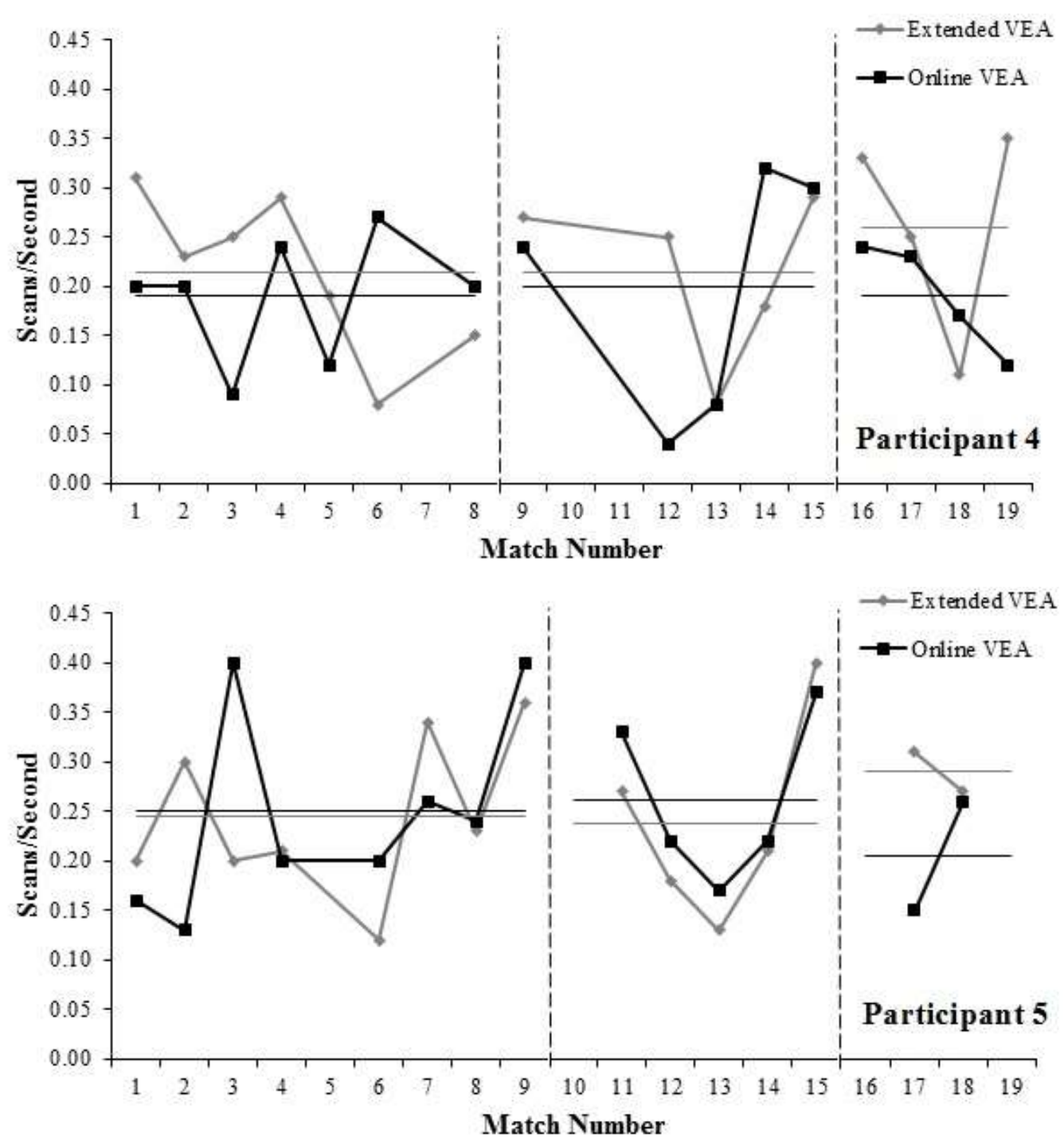

304

Figure 1: Pre- and post-intervention VEA in the extended and online scanning periods ${ }^{\mathrm{a}}$.

${ }^{a}$ Missing data points are due to injury or non-selection. Participant 4 sustained a minor injury during the intervention period and the intervention was prolonged to seven weeks for this participant. 

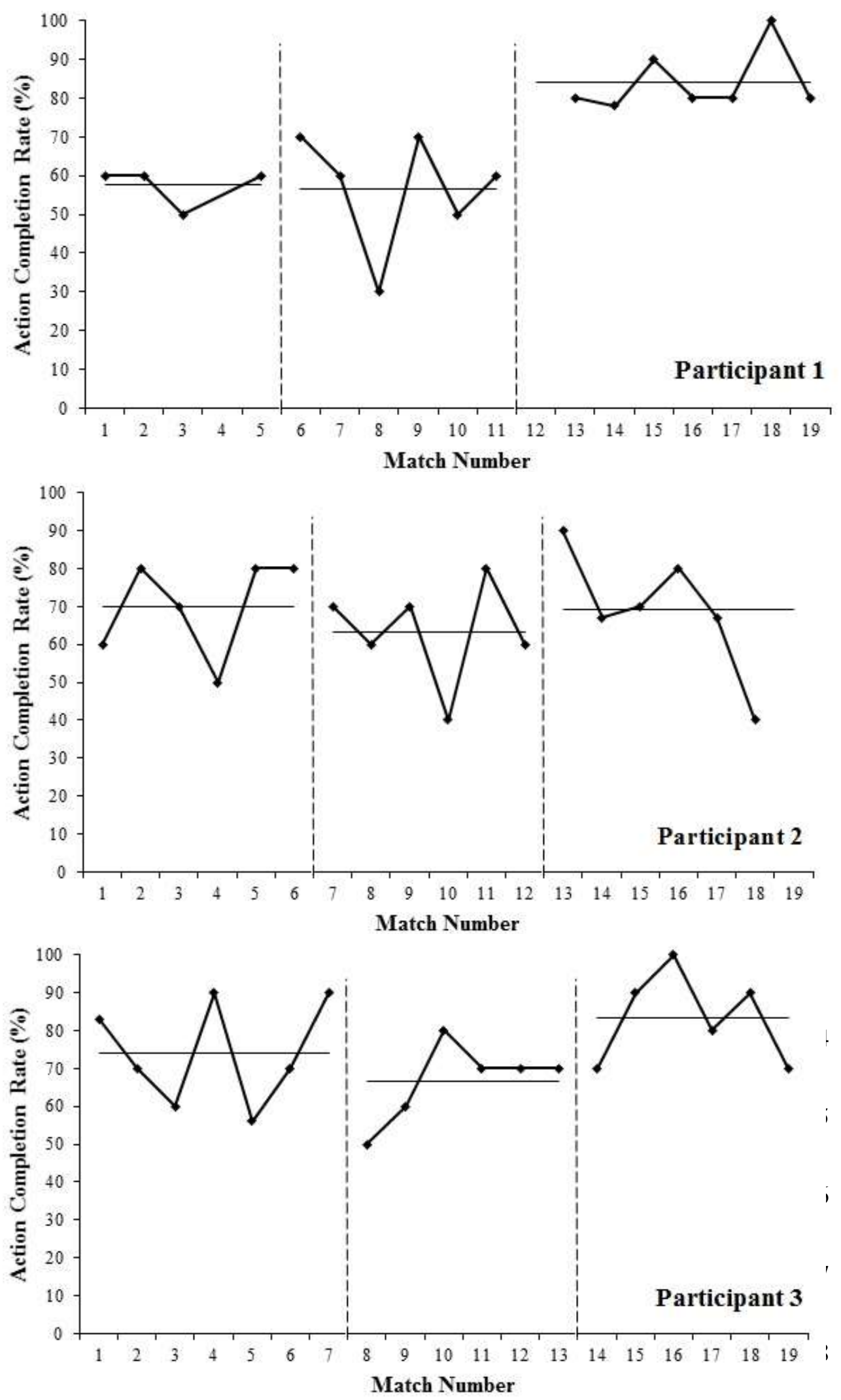

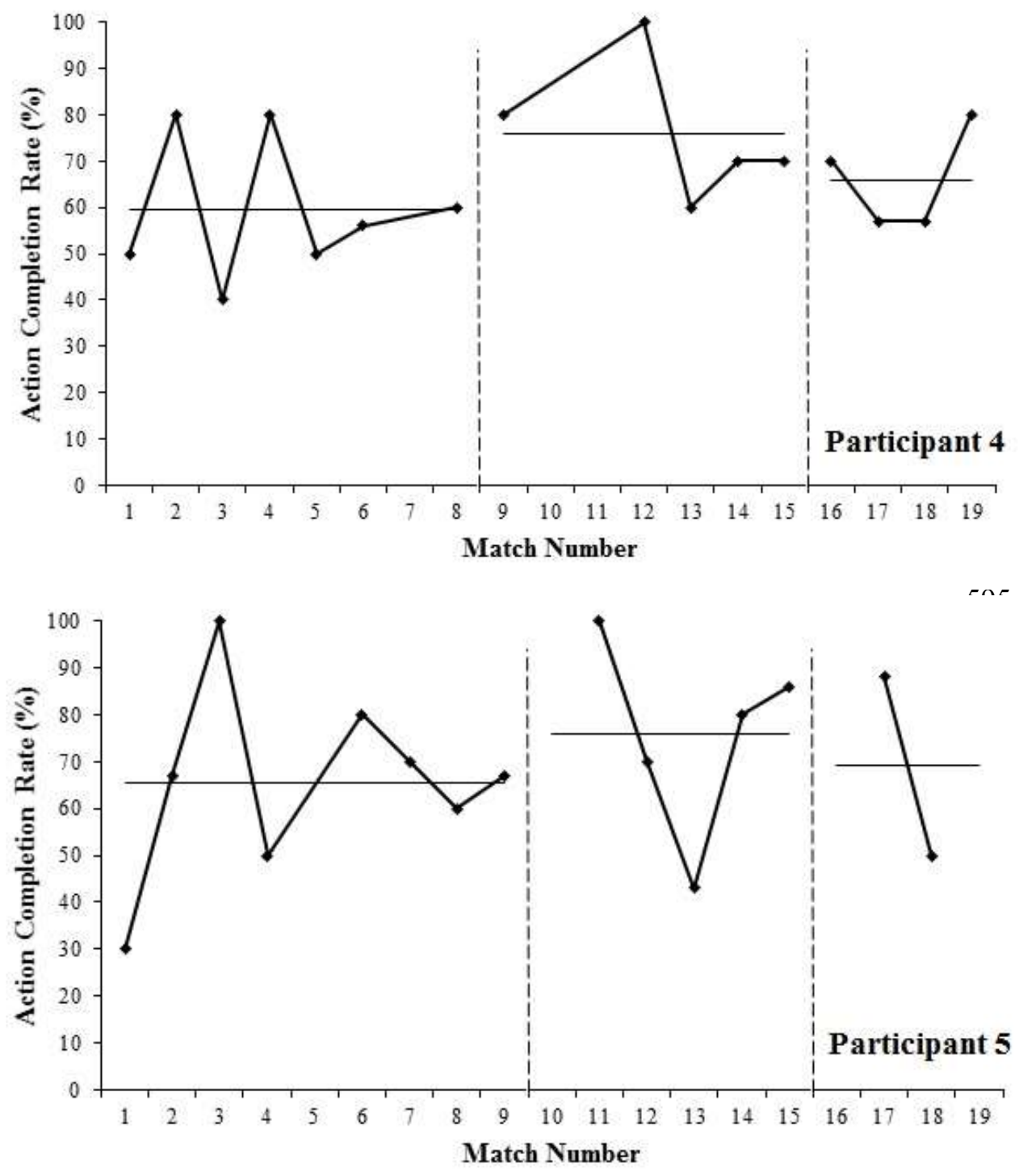

Figure 2: Pre- and post-intervention action completion rates.

603

604

605 
607

608

609

610

611

612

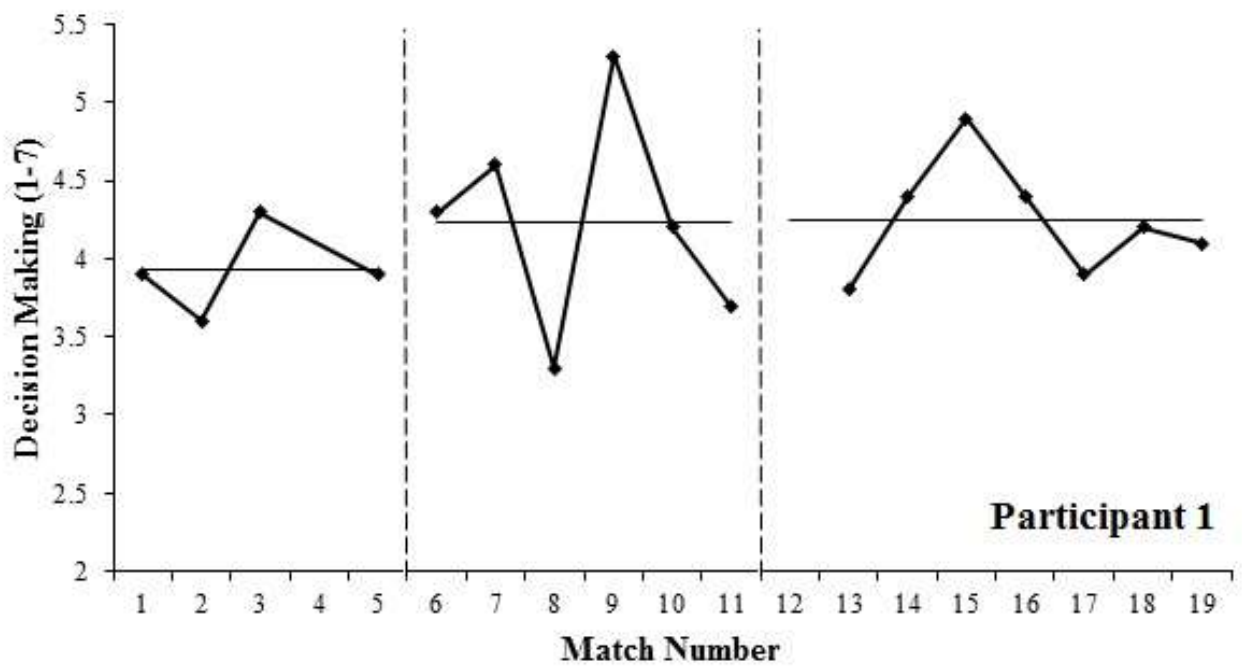

613

614

615

616

617

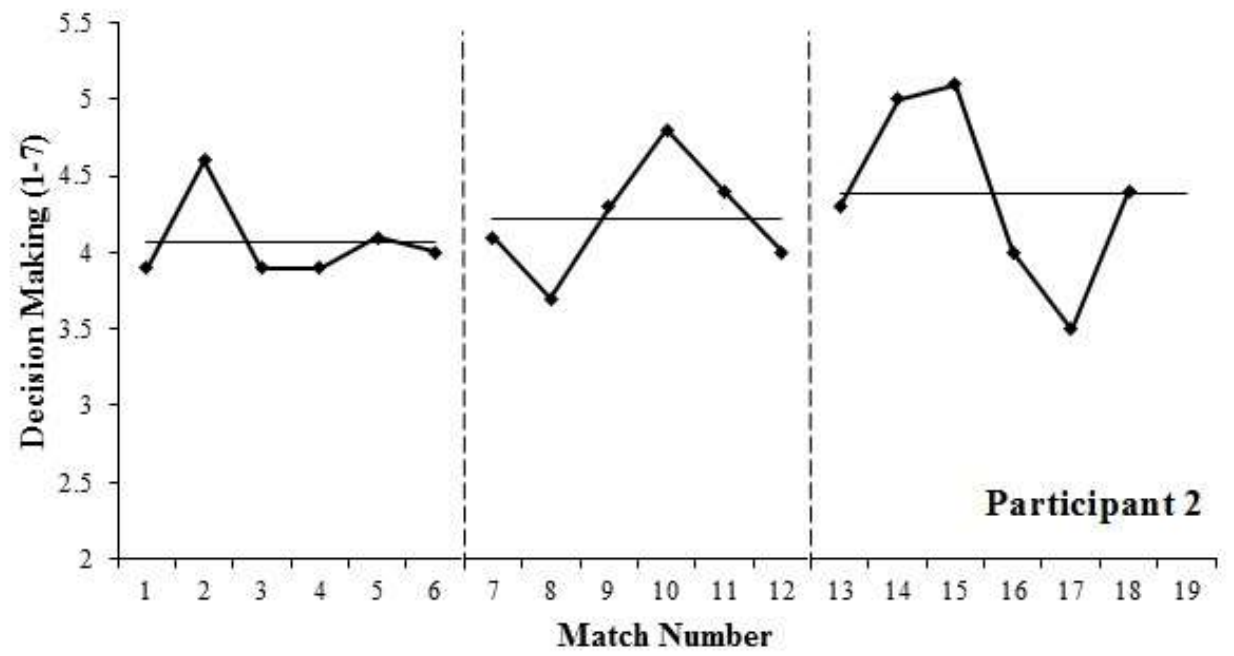

618

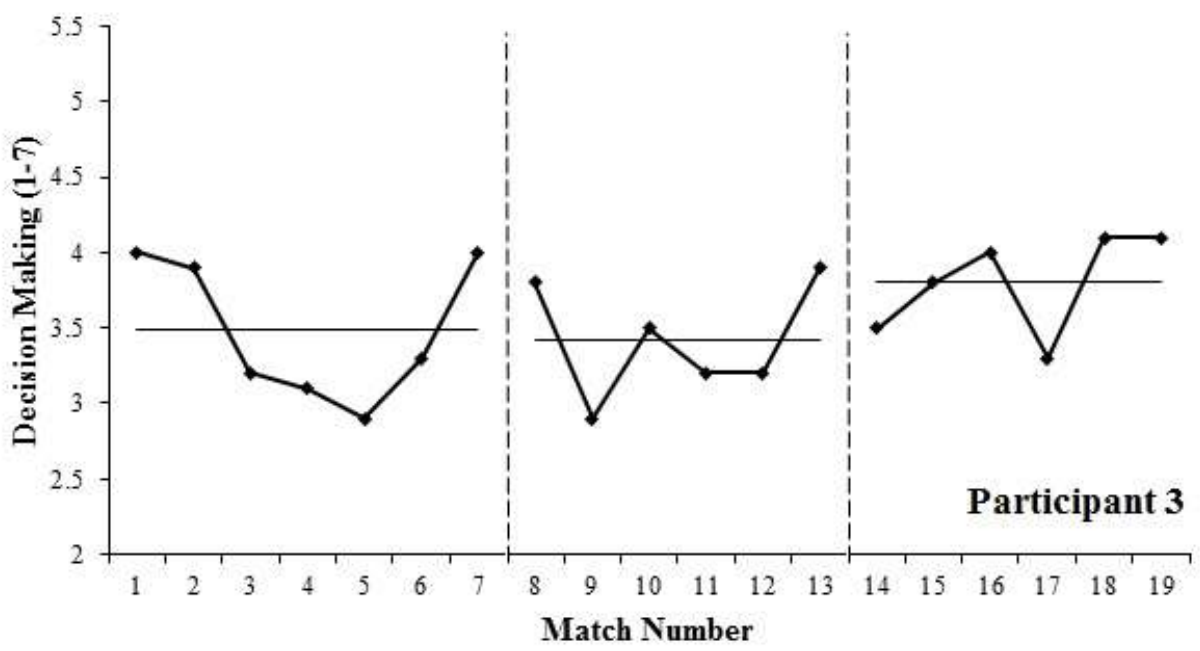


623

624

625

626

627

628

629

630

631

632

633

634

635

636

637

638

639

640
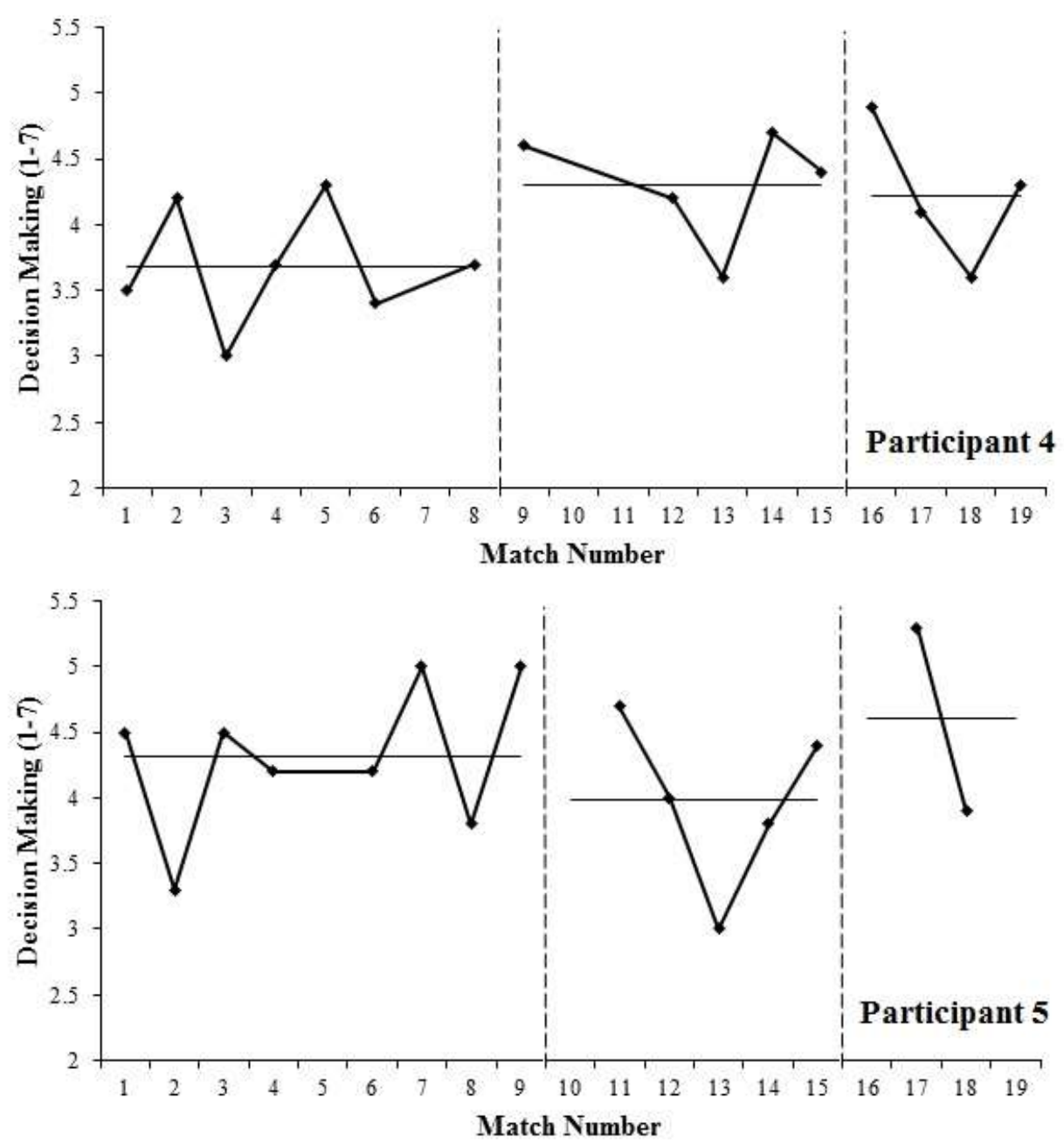

Figure 3: Pre- and post-intervention decision-making scores. 
641 Table 1: Baseline and post-intervention imagery ability scores.

642

Baseline

Post-Intervention

643

\begin{tabular}{lllllllll}
\hline & \multicolumn{2}{c}{ Visual } & Kinesthetic & \multicolumn{2}{l}{ Visual } & \multicolumn{2}{l}{ Kinesthetic } \\
& & & & & & \\
\hline Participant & $M$ & $S D$ & $M$ & $S D$ & $M$ & $S D$ & $M$ & $S D$
\end{tabular}

644

\begin{tabular}{lllllllll}
\hline 1 & 6.00 & 0.00 & 3.00 & 0.82 & 6.25 & 0.50 & 5.50 & 0.58
\end{tabular}

645

$\begin{array}{lllllllll}2 & 4.75 & 0.50 & 5.25 & 0.50 & 5.25 & 0.96 & 5.25 & 0.50\end{array}$

646

647

3

$2.00 \quad 0.82$

2.75

1.26

5.50

0.58

5.50

0.58

4

$\begin{array}{llll}4.75 & 0.50 & 3.00 \quad 0.82\end{array}$

5.75

0.50

5.75

0.50

648

5

$6.00 \quad 0.00$

$4.25 \quad 0.96$

6.75

0.50

$6.50 \quad 0.58$ 